\title{
An Evaluation on Oral Vestibular Schwannoma Growth from the View Point of Molecular Biology
}

\author{
Bing H Tang* \\ Master of Public Health, Columbia University, USA \\ *Corresponding author: Bing H Tang, Master of Public Health, Columbia University, 116th St Broadway, New York, USA
}

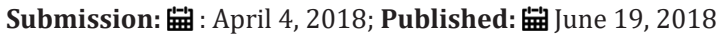

\section{Opinion}

This author has known Medical Hypotheses several years ago, unfortunately did not know that it is available online lately, until recently when he read Prof. Gong Peng's important hypotheses. Has been honored to receive the invitation from Prof. Ping Gong [an oral surgeon] for our potential collaboration. In the meantime, please correct some possible errors in this author' writing as presented below, and the possibility of applying his own concepts into the important future research.

\section{Vestibular Schwannoma Growth}

This author read with great interest the article written by Baser et al. [1]. (Baser ME, Makariou EV, Parry DM: Predictors of vestibular schwannoma growth in patients with neurofibromatosis Type 2. J Neurosurg 96:217-222, February, 2002).

Their research expresses result of two longitudinal studies of growth rates of vestibular schwannomas (VSs) in patients with neurofibromatosis Type 2 (NF2) differs from each other as to whether VS growth rates decrease or increase when ever patients getting older. The authors undertook their study to assess the relationship between VS growth rates and patient age and type of constitutional NF2mutation; they also examined the in VS growth rates among multiple patients in families with NF2. Methods by using Gadolinium-enhanced magnetic resonance images obtained in 18 patients with inherited NF2 from 11 unrelated families whom were retrospectively analyzed. The patients had been observed for a period with a median of 4 years. Volumes of the VSs were checked using a two component box model (intrameatal and extrameatal parts checked respectively). Single strand conformation polymorphism analysis and Southern blot analysis were employed to constitutional NF2mutations. Growth rates of the VSs were highly flexible but have a tendency to diminution with increasing patient age both at commencement of signs or symptoms of NF2 ( $\mathrm{r} 2=0.35, \mathrm{p}=0.026)$ and at diagnosis ( $\mathrm{r} 2=0.33$, $\mathrm{p}=0.012$ ). The VS growth rates did not fluctuate significantly with the type of constitutional NF2mutation or the number of non-VS either cerebral or spinal tumours. The VS growth rates were highly modifiable within families, but, did not correspond to clinical pictures of NF2 disease severity, such as patient age at the symptom commencement and the actual numbers of non VS cerebral and spinal tumours respectively Tang B [2] .

Conclusions of their study can be summarized as follow-the growth rates of VSs in patients with NF2 are highly variable but tend to decrease with increasing patient age. Clinical treatment of multiple patients in families with NF2 cannot be based on the expectations of similar VS growth rates, even when other clinical aspects of disease severity are so close to each other, the latter including other nervous system tumours' occurrence rate.

Being due to the fact that vestibular schwannomas also develop sporadically, and the other fact of their onset usually takes place when the patient is older [1]. Age per se, however, does not explain the detected difference in proliferation of tumours. One of the explanations is that VSs in younger patients with NF2 also had higher MIB-1 indices (Ki-67; monoclonal antibodies) than their counterparts, sporadic tumours' in age-matched patients; this is why MIB-1 monoclonal antibody testing is so much useful. It is also very interesting and important that within spinal tumours', malignant peripheral nerve sheath tumours' have higher MIB-1 pictures.

Though the defective NF2gene is important in the tumorigenesis of both NF2 and sporadic schwannom as, the differences in the molecular biology of these tumours, rather than any difference in ages among the patients are crucial [2]. This author certainly agree with the authors that there are marked interfamily differences in disease severity and tumour susceptibility in NF2. 4 In fact, larger acoustic neuromas were more commonly found in patients with concomitant spinal tumours' or meningiomas. Patients with NF2 with spinal tumours' but not meningiomas demonstrated faster growth rates than patients without any additional tumour in another part of the nervous system. Analysis of the data from the study by Abaza et al. [3] indicates that patient with associated spinal tumours' have faster growing acoustic neuromas and therefore should be followed closely and treated even more aggressively.

Any allocation of patients with VS to the so-called wait-and-see treatment policy, in my opinion, must be more thoroughly assessed and revaluated [3]. 
With regard to molecular biology, paying special attention to the genotype-phenotype correlation in NF2, we now know that the splice-site mutations in NF2 are associated with various phenotypes, which range from severe to symptom free [4]. In addition to interfamily differences, phenotypic variations are also observed within families [5]. Mutations downstream from exon 8 (in contrast to intron, extron is a segment of a gene that is decoded to provide a messenger RNA or mature RNA product) result more often in mild phenotypes of NF2. This indicates that splice-site alteration is a relatively common cause of NF2, and that splicesite alteration does not look like any other mutations; the clinical outcomes of splice-site mutations in the NF2gene are quite variable [6]. For this very reason, the tremendously growing volume of information on genotype-phenotype correlation in NF2 has been enlarged and expanded [7].

On the other hand, the missense mutation (that is, a nucleotide substitution that results in an amino acid change) does take place in patients with mild phenotypes, in contrast to the splice-site mutation, which occurs in families with both mild and severe phenotypes of NF2 Antinheimo J [4]. It is worthwhile to note that truncating mutations of NF2are usually associated with severe phenotypes, but the association of some mutations with mild and severe phenotypes indicates that NF2 expression is affected by stochastic, epigenetic, or environmental factors Charabi [5]. In my professional opinion, and with a reasonable degree of medical certainty, mental factors (as in the spiritual dimension) might also play a role [8]. Last but not the least, in a larger study of 132 unrelated families reported by Evans et al. [6]. The authors, Evans et al have successfully confirmed the previous impression that truncating mutations are associated with a severe form of NF2 Trueman L [7]. Nonetheless, due clinical sentences are still needed to be exercised to use mutation types for predicting the disease course of NF2 Baser \& Kluwe L [1]

This author encourages Baser et al. [1] for their fine quality of their work, and sincerely hope that in the near future they can find any other possible predictors of VS growth rates in patients with NF2 Tatagiba M [8].

\section{References}

1. Kluwe L, Bayer S, Baser ME, Hazim W, Haase W, et al. (1996) Identification of NF2 germline mutations and comparison with neurofibromatosis 2 phenotypes. Hum Genet 98(5): 534-538.

2. Tang B (2004) Predictors of Vestibular Schwannoma Growth in patients with Neurofibromatosis Type 2. J of Neurosurgey 100: 734.

3. Abaza MM, Makariou E, Armstrong M, Lalwani AK (1996) Growth rate characteristics of acousticneuromas associated with neurofibromatosis type 2. Laryngoscope 106(6): 694-699.

4. Antinheimo J, Haapasalo H, Seppala M, Sainio M, Carpen O, et al. (1995) Proliferative potential of sporadic and neurofibromatosis 2-associated schwannomas as studied by MIB-1 (Ki-67) and PCNA labeling. J Neuropathol Exp Neurol 54(6): 776-782.

5. Charabi S, Thomsen J, Mantoni M, Charabi B, Jorgensen B, et al. (1995) Acoustic neuroma (vestibular schwannoma): growth and surgical and nonsurgical consequences of the wait and see policy. Otolaryngol Head NeckSurg 113(1): 5-14.

6. Evans DG, Huson SM, Donnai D, Neary W, Blair V, Newton V, et al. (1992) A clinical study of type 2 neurofibromatosis. Q J Med 84(304): 603-618.

7. Evans DG, Trueman L, Wallace A, Collins S, Strachan T (1998) Genotype/ phenotype correlations in type 2 neurofibromatosis (NF2): evidence for more severe disease associated with truncating mutations. J Med Genet 35(6): 450-455.

8. Kluwe L, MacCollin M, Tatagiba M, Thomas S, Hazim W, et al. (1998) Phenotypic variability associated with 14 splice site mutations in the NF2gene. Am J Med Genet 77(3): 228-233.
Creative Commons Attribution 4.0 International License

For possible submissions Click Here

\section{Submit Article}

